\title{
Design optimization and experimental analysis of Piezoelectric Energy harvester
}

\author{
A. Rami Reddy, D. Ezhilarasi, M. Umapathy \\ Department of Instrumentation and Control Engg. National Institute of Technology, Tiruchirappalli-620015, \\ India
}

\begin{abstract}
This paper describes an approach to harvest electrical energy from a mechanically excited piezoelectric element. The structure of piezoelectric energy harvester is optimized for maximum power with minimum dimensions through Genetic Algorithm approach to enhance the conversion of mechanical energy into electrical energy using direct piezoelectric effect. Numerical analysis is carried out to obtain optimal PZT position on the cantilever beam to get maximum harvested power. A rectifier with boost converter IC is used for converting harvested power in to usable DC power. The simulation results are verified experimentally.
\end{abstract}

Keywords: Energy harvester, FEM analysis, Genetic Algorithm, Optimization, Piezoelectric.

\section{Introduction}

Over the last couple of decade energy harvesting technologies have received a great attention at both the industrial and academic levels. Harvesting energy from the environment (solar, thermal, kinetic, etc.) is a promising alternative to batteries and it has been receiving much more attention lately due to the increasing demand for wireless sensor networks, implantable medical electronics, tire-pressure sensor networks, MEMS devices etc.[1]-[6]. Different energy harvesting techniques have been investigated for the purpose to broaden the energy sources, alleviate the dependence on batteries, and hopefully someday make all WSN devices selfpowered[7]-[12].

There are various methods to convert ambient energy into electrical energy. According to the conversion mechanism, the power harvesting device can be classified into three types: piezoelectric, electromagnetic and electrostatic [13]. Among these different types of conversion, piezoelectric transducers have been of great promise and interest. In an energy harvesting system using piezoelectric materials, mechanical energy can be converted to electrical energy through the piezoelectric effect, and the wasted energy such as wind and tidal energy and the generated vibrations on the structure will be reutilized [14]. Vibration is one of the most commonly available forms of ambient energy, found in civil structures, machines, the human body, etc. Various vibration sources with their maximum acceleration magnitude and frequency range were well listed by Roundy et al. [15]-[19] and it is a popular way of extracting electrical energy from the environment [20]-[29].

In this work the analytical design of energy harvesting structure is presented and the same is optimized using Genetic Algorithm to get maximum power. Numerical optimization is done using the COVENTORWARE to fix the PZT position on the beam from the fixed end. With the optimized design, experimentation is carried out with magnetic force induced vibration to give maximum output power in $\mathrm{mW}$.

\section{Analytical design of Energy harvesting structure}

The piezoelectric energy harvester is described by two PZT layers mounted on an Aluminum rectangular cantilever beam with a proof mass placed at the free end, as shown in Fig. 1. In order to vibrate the cantilever beam, one cylindrical permanent magnet is attached at the free end of the cantilever beam (tip mass) and other one is cylindrical electromagnet. The tip is excited through an electromagnet placed above at a distance $x_{1}$. The electromagnet is a type of magnet in which the magnetic field is produced by the flow of electric current. The magnetic field disappears when the current is turned off. Much stronger magnetic fields can be produced if a "core" of ferromagnetic material, such as soft iron, is placed inside the coil. The magnetic field created by the electromagnet is proportional to both the number of turns in the winding, $N$, and the current in the wire, $i$ hence this product, $N i$, in ampere-turns, is the name magnetomotive force. The complete analytical model of the energy harvester along with electromagnet is presented below. 
Top PZT-5H with length 1 , width $\mathrm{b}$, thickness $\mathrm{t}$
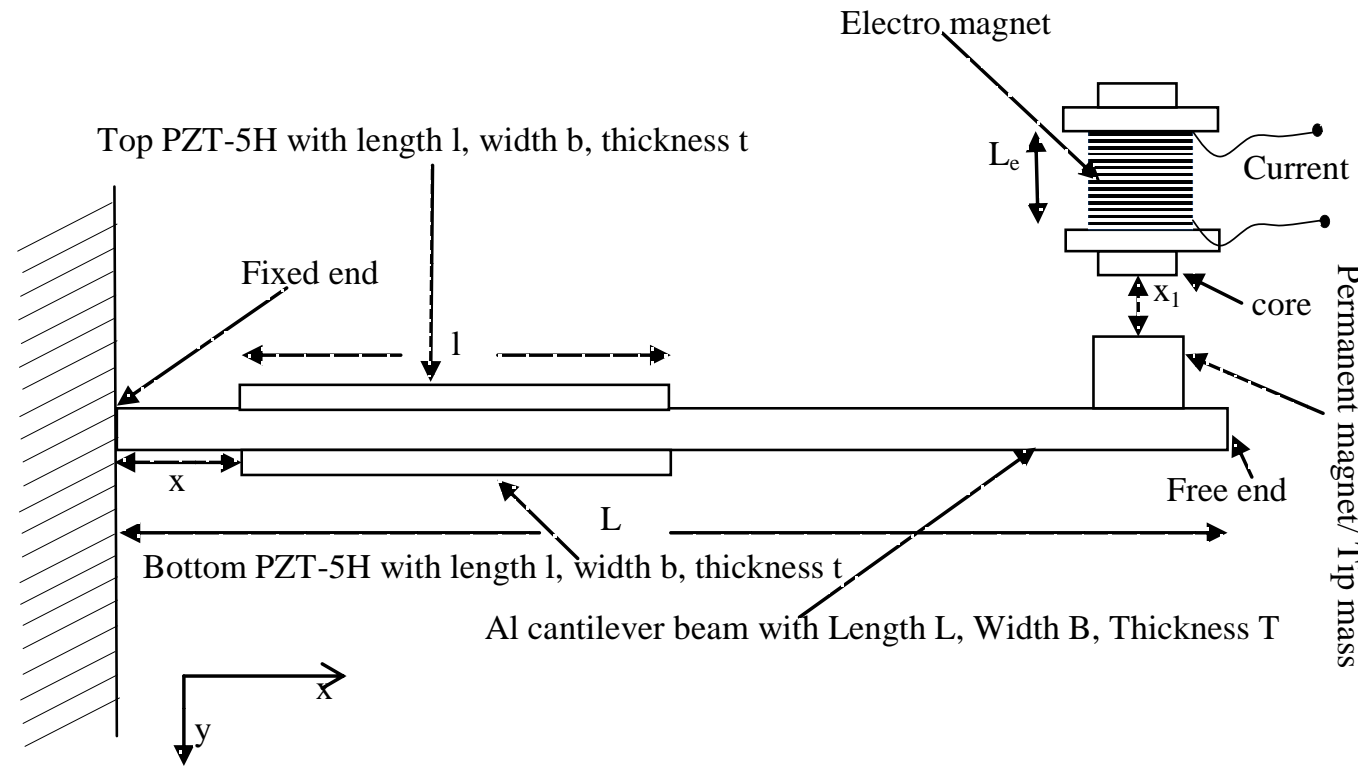

Fig. 1 The schematic of the piezoelectric energy harvester

The inductance of the coil (electromagnet) is, $\quad L_{c}=\frac{\mu N^{2} A}{L_{e}}$

Where ' $\mu$ ' is the permeability of the iron core, ' $N$, is the number of turns in the coil, ' $A$ ' is the cross sectional area of core and ' $L_{e}$ ' is the length of the electromagnet. The power given by the electromagnet is,

$$
P_{i}=\frac{1}{2} L_{c} i^{2}
$$

where, ' $i$ ' is alternating current through the electromagnet.

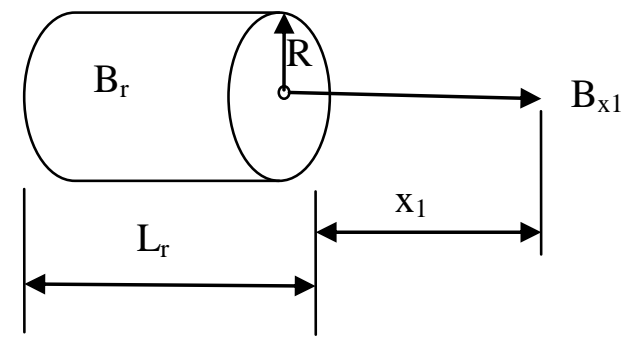

Fig. 2 Magnet flux density at point $\mathrm{x}_{1}$

The magnetic flux density at a distance ' $x_{1}$ ' from the cylindrical permanent magnet, from Fig. 2 is,

$$
B_{x_{1}}=\frac{B_{r}}{2}\left(\frac{L_{r}+x_{1}}{\sqrt{R^{2}+\left(L_{r}+x_{1}\right)^{2}}}-\frac{x_{1}}{\sqrt{R^{2}+x_{1}^{2}}}\right)
$$

where, ' $L_{r}$ ', ' $R$ ' and ' $B_{r}$ ' are the length, radius and flux density of the permanent magnet respectively. The alternating force between the permanent magnet and the electromagnet is, $F_{\text {current }}=N i A B_{x_{1}} / L_{e}$

From, Eqs. (3) and (4), $F_{\text {current }}=\frac{N i A B_{r}}{2 L_{e}}\left(\frac{L_{r}+x_{1}}{\sqrt{R^{2}+\left(L_{r}+x_{1}\right)^{2}}}-\frac{x_{1}}{\sqrt{R^{2}+x_{1}^{2}}}\right)$

The Eq. (5) is only an approximation which does not consider hysteresis between the magnetic force of attraction and repulsion. The Eq. (1) - (5) are used to estimate force applied to the free end of the beam. The approximate stress and deflection at specific point ' $x$ ' as a function of ratio of distance from the fixed end to the specific location on beam is obtained from Hooke's law. 
Stress is given by, $\sigma=\frac{F_{\text {current }}}{z}(L-x)$

Where ' $L$ ' is the Length of the cantilever beam, ' $z$ ' is the section modulus, $\quad z=\frac{B T^{2}}{6}$

Where ' $B$ ' is the width of the beam, ' $T$ ' 'is the thickness of the beam.

Deflection at specified point $x$, is given by, $y=\frac{F_{\text {current }} x^{2}}{6 E I}(3 L-x)$

where, ' $E$ ' is the modulus of elasticity of beam material, ' $I$ ' is the moment of inertia, $I=\frac{B T^{3}}{12}$

The resonant frequency is found by combining Newton's Second Law and Hooke's Law.

Resonance frequency $\omega=\sqrt{\frac{E B T^{3}}{4(M+m) L^{3}}}$

where, $M$ is the mass of the structure, $m$ is the mass of the permanent magnet(tip mass).

If piezoelectric patches are bonded at certain section of the beam surface, the output voltage from PZT can be estimated by multiplying the stress at that location with the piezoelectric voltage constant, $g_{31}$. Assuming that the material is linear, elastic, and isotropic with an average stress applied along the $1-1$ direction, the output voltage can be determined as follows:

$V=g_{31} * \sigma * t$

where, ' $t$ ' is the thickness of the piezoelectric material. Combining Eqs. (5), (6) and (7), we get,

$\sigma=\frac{6(L-x)}{B T^{2}} \frac{N i A B_{r}}{2 L_{e}}\left(\frac{L_{r}+x_{1}}{\sqrt{R^{2}+\left(L_{r}+x_{1}\right)^{2}}}-\frac{x_{1}}{\sqrt{R^{2}+x_{1}^{2}}}\right)$

Combining Eqs. (11) and (12), we get,

$V=3 g_{31} t \frac{(L-x)}{B T^{2}} \frac{N i A B_{r}}{L_{e}}\left(\frac{L_{r}+x_{1}}{\sqrt{R^{2}+\left(L_{r}+x_{1}\right)^{2}}}-\frac{x_{1}}{\sqrt{R^{2}+x_{1}^{2}}}\right)$

Resistive load for which the output power reaches its maximum valve is given by (Lu et al).

Resistive Load, $R_{L}=\frac{t}{B L \varepsilon_{33} w}$

where, ' $\varepsilon_{33}$ ' is the relative permittivity of piezoelectric material, ' $w$ ' is the width of the PZT.

From Eqs. (13) and (14), the output power of the piezoelectric energy harvester connected to a resistive load can

be expressed as: $P_{o}=\frac{V^{2}}{R_{L}}$

From the Eqs. (2) and (15), the efficiency of the piezoelectric energy harvester is calculated.

\section{Optimization of structure using Genetic Algorithm}

In their most basic form, Genetic Algorithm's are function optimizers, i.e., methods for seeking extreme of a given objective function. Here the design objective is to get maximum power from minimum structure and PZT dimension. Using the analytical design presented in section 2 the energy harvesting structure is optimized by Genetic Algorithm. The four design constraints used are as follows. The Maximum volume of the Beam and PZT are $12000 \mathrm{~mm}^{3}$ and $1000 \mathrm{~mm}^{3}$ respectively, the minimum power to be $5 \mathrm{~mW}$ and maximum stress is $5 \mathrm{MNm}^{-2}$. The properties of the piezoelectric energy harvester are shown in Table 1. 


\begin{tabular}{ll}
\multicolumn{2}{c}{ Table 1: Properties of Energy harvester } \\
\hline Property & Value \\
\hline Residual flux density of the permanent magnet $\left(B_{r}\right)$,Tesla & 1.1 \\
Length of the permanent magnet $\left(L_{r}\right), \mathrm{mm}$ & 6.5 \\
Radius of the permanent magnet $(R), \mathrm{mm}$ & 4.5 \\
Length of the coil $\left(L_{e}\right), \mathrm{mm}$ & 16 \\
Number of turns in coil $(N)$ & 2000 \\
Young's modulus of the beam $(E), \mathrm{Gpa}$ & 71 \\
Piezoelectric strain constant $\left(d_{31}\right), \mathrm{mV}^{-1}$ & $-247 \times 10^{-12}$ \\
Piezoelectric stress constant $\left(g_{31}\right), \mathrm{VmN}^{-1}$ & $-9 \times 10^{-3}$ \\
Dielectric constant $\left(\mathrm{K}_{3}^{\mathrm{T}}\right)$ & 3100 \\
Modulus of elasticity of $\mathrm{PZT}(\mathrm{E}), \mathrm{Gpa}$ & 47.62 \\
Density of PZT $\left(\rho_{\mathrm{PZT}}\right), \mathrm{Kgm}{ }^{-3}$ & 7500 \\
Density of Al beam $\left(\rho_{\mathrm{Al}}\right), \mathrm{Kgm}^{-3}$ & 2700 \\
Relative permittivity of piezoelectric material $\left(\varepsilon_{33}\right), \mathrm{Fm}^{-1}$ & $3500 \times 8.85 \times 10^{-12}$ \\
Distance between magnets $\left(\mathrm{x}_{1}\right), \mathrm{mm}$ & 6 \\
Radius of the core of the electromagnet $(\mathrm{R}), \mathrm{mm}^{-1}$ & 6.5 \\
permeability of iron core $(\mu), \mathrm{Hm}{ }^{-1}$ & $8.5 \times 10^{-3}$ \\
permanent electromagnet $(\mathrm{tip})$ mass $(\mathrm{m}), \mathrm{Kg}$ & $7.8 \times 10^{-3}$ \\
Mass of the structure $(\mathrm{M}), \mathrm{Kg}^{-3}$ & $29 \times 10^{-3}$ \\
\hline
\end{tabular}

The Genetic Algorithm is an iterative procedure which maintains a constant-size population of candidate solutions. During each iteration step, called a generation, the structures in the current population are evaluated, and, on the basis of those evaluations, a new population of candidate solutions is formed. The parameters defined in Genetic Algorithm are as follows: the number of strings, number of generations/iterations and string populations are taken as 100 and number of parents is assumed to be 10 . The analysis for optimum dimensions of the piezoelectric energy harvester for the given constraints is carryout. The Results of the Genetic Algorithm Optimization Method are shown in Table 2.

Table 2: Optimum dimensions of Energy harvester from Genetic algorithm

\begin{tabular}{llll}
\hline Parameter & Lower limit & Upper limit & Optimum value from GA \\
\hline Length of the Beam(L),mm & 70 & 200 & 120 \\
Width of the beam(B),mm & 10 & 30 & 25 \\
Thickness of the beam(T),mm & 0.5 & 2 & 1.2 \\
Length of PZT(1),mm & 70 & 80 & 76 \\
Width of PZT(b),mm & 20 & 30 & 25 \\
Thickness of PZT(t),mm & 0.1 & 1 & 0.5 \\
\hline
\end{tabular}

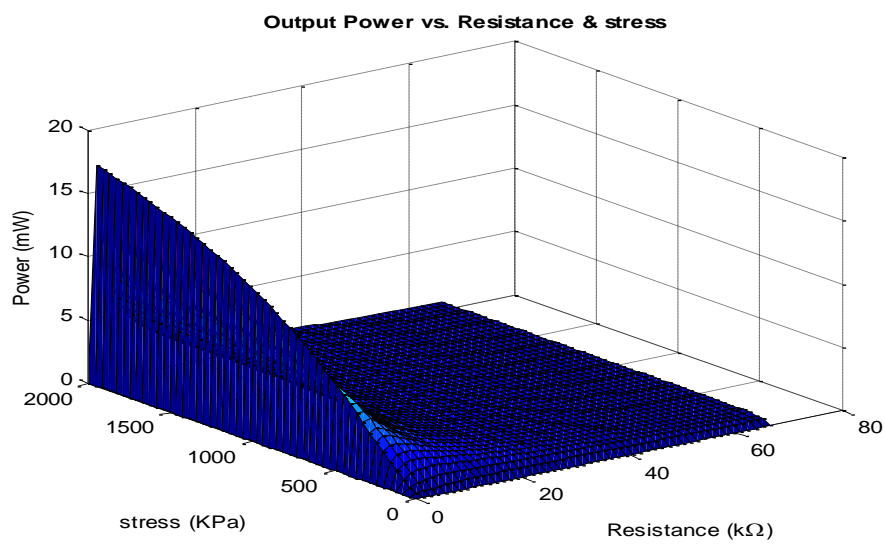

Fig. 3 Output power as function of both external resistance and stress from GA

The optimum beam volume, PZT volume and power obtained are $3600 \mathrm{~mm}^{3}, 950 \mathrm{~mm}^{3}$ and $17 \mathrm{~mW}$ respectively for the stress of $2 \mathrm{Mpa}$. Output power as function of both external resistance and stress for the design parameters 
obtained from the genetic algorithm is shown in Fig. 3. From the results it is observed that the power increases when stress increases and resistance decreases.

\section{Numerical design and analysis for optimum PZT position on cantilever beam}

For the optimum dimension of Energy harvester obtained from Genetic Algorithm, the maximum output power varies with respect to the PZT position on the beam. Since the bending moment along the beam as well as generated voltage from PZT varies with respect to the composite element position on the beam from the fixed end, numerical design is carried out using FEM design and simulation software COVENTORWARE. The process steps required to design Energy harvester are shown in Fig. 4. Fig. 5 shows the layout for building the solid model of the Energy harvester. For mapping the model, non-linear Tetrahydron element of element size $0.1 \mathrm{~mm}$ is used. One end of the beam is taken as fixed for the fixed end of the beam. The other end of the beam a mass is attached as a tip mass to load the beam for vibration. The PZT 5H (Piezoceramic) is attached to the beam and the generated voltage measured from electrodes. The developed solid 3-dimension model of the Energy harvester with the required mesh for the finite element analysis is shown in Fig. 6.

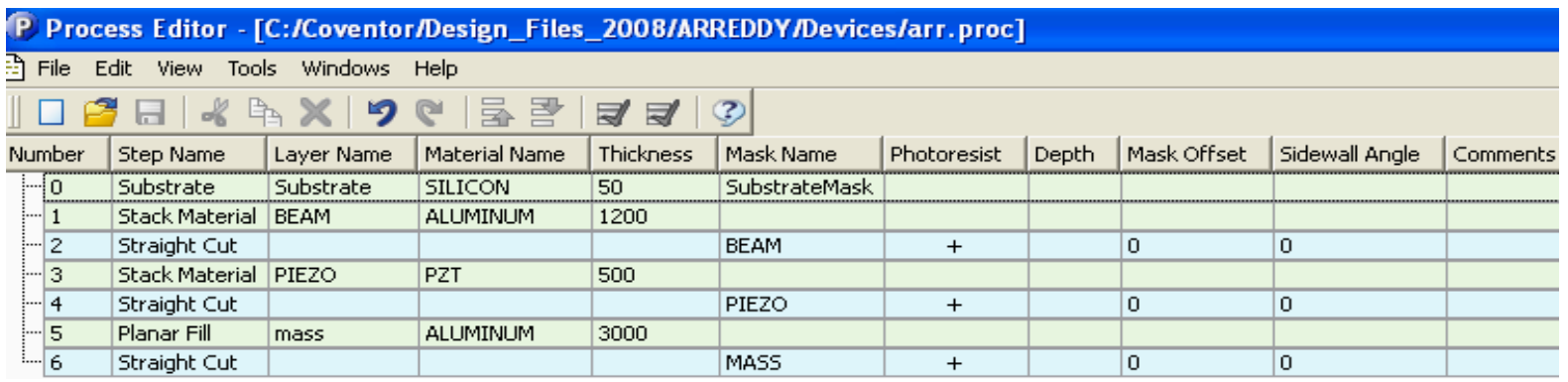

Fig. 4 Process steps of the Energy harvester
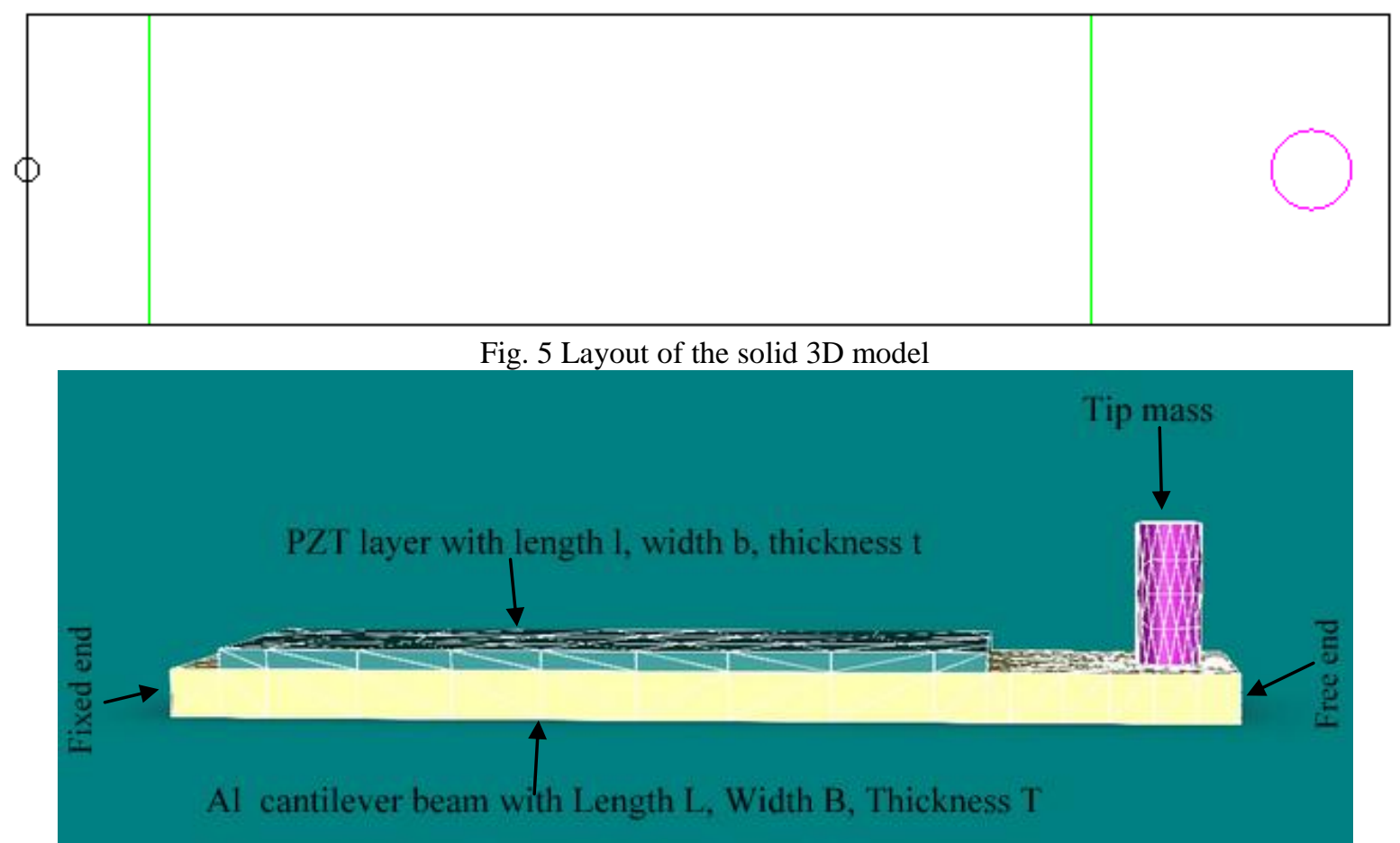

Fig. 6 The solid 3D model of the Energy harvester

The optimum dimensions of PZT and Aluminum cantilever beam obtained from Genetic Algorithm are used in numerical analysis. The PZT position on the cantilever beam from the fixed end for maximum output is analyzed through MemMech piezoelectric analysis. Using the modal analysis the first mode resonance frequency is analyzed for each PZT position on the Al cantilever beam from the fixed end which is shown in Fig. 7. From the results it is found that the resonance frequency decreases when the piezoceromic composite element moved from the fixed end. 


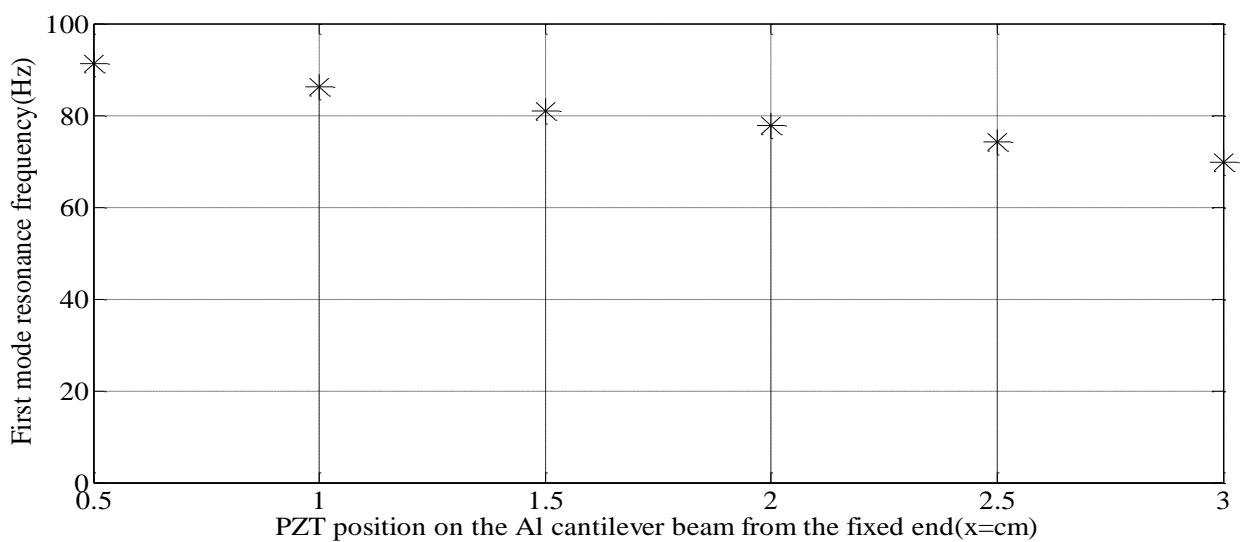

Fig. 7 First mode resonance frequencies corresponding to each PZT position on the Al cantilever beam from the fixed end

Simulation is carried out using direct harmonic analysis at first mode resonance frequency with a load of $0.001 \mathrm{~N}$ for different PZT positions which is shown in Fig. 8. The variable ' $\mathrm{x}$ ' represents the PZT position on the Al cantilever beam from the fixed end. From the results it is seen that the maximum voltage of $1.2 \mathrm{~V}$ is produced by the piezoelectric element when it is placed at a distance of $2 \mathrm{~cm}$ from the fixed end, which is three times greater than that with piezo element at fixed end.

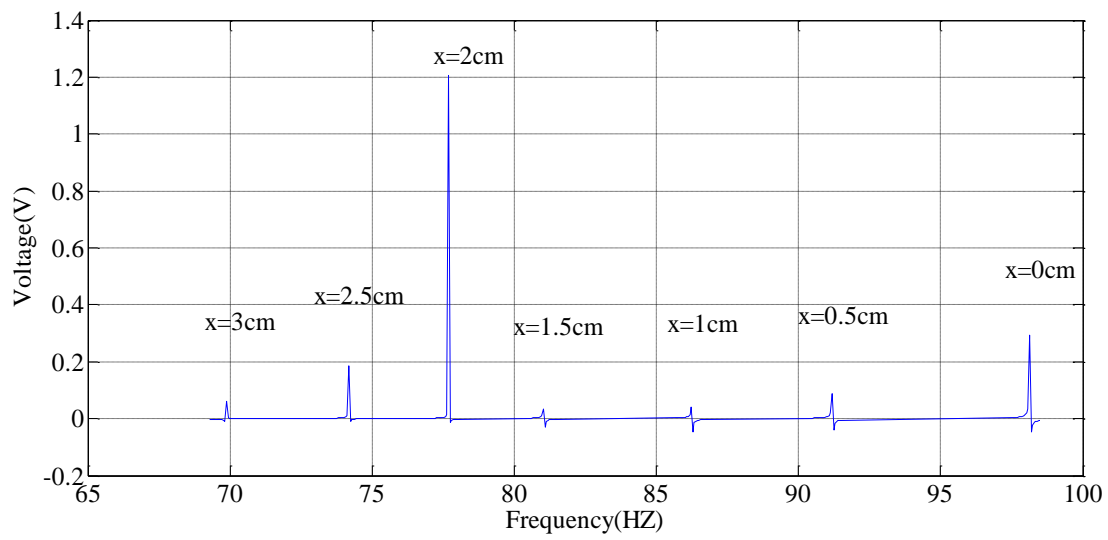

Fig. 8 The voltage output for different PZT positions from the fixed end of the cantilever beam at first mode resonance frequencies.

Similar kind of analysis (VARY analysis) is also carried out using Architect to get the frequency response for different PZT positions directly. The schematic created in Architect is shown in Fig. 9. The Al cantilever beam is fixed at one end. A load of $0.001 \mathrm{~N}$ is applied at the free end. The PZT position on the Al cantilever beam is varied from the fixed end using VARY analysis. At each PZT position resonance frequency and the corresponding voltage output is found using small signal ac analysis. Fig. 10 shows frequency versus voltage output at different PZT positions on the Al cantilever beam from the fixed end. From the above numerical analyses the optimum PZT location from the fixed end of the cantilever beam for maximum voltage output is found to be $2 \mathrm{~cm}$.

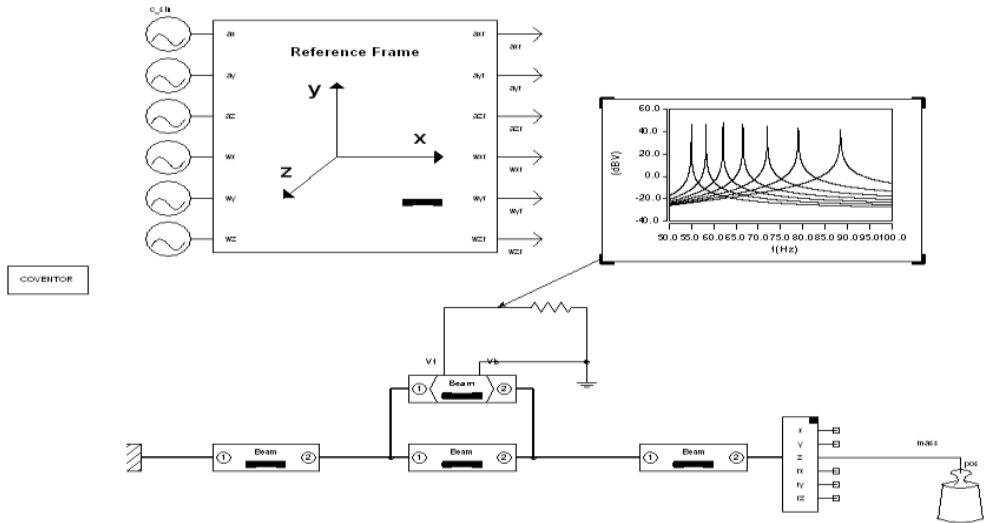

Fig. 9 The schematic created in Architect 


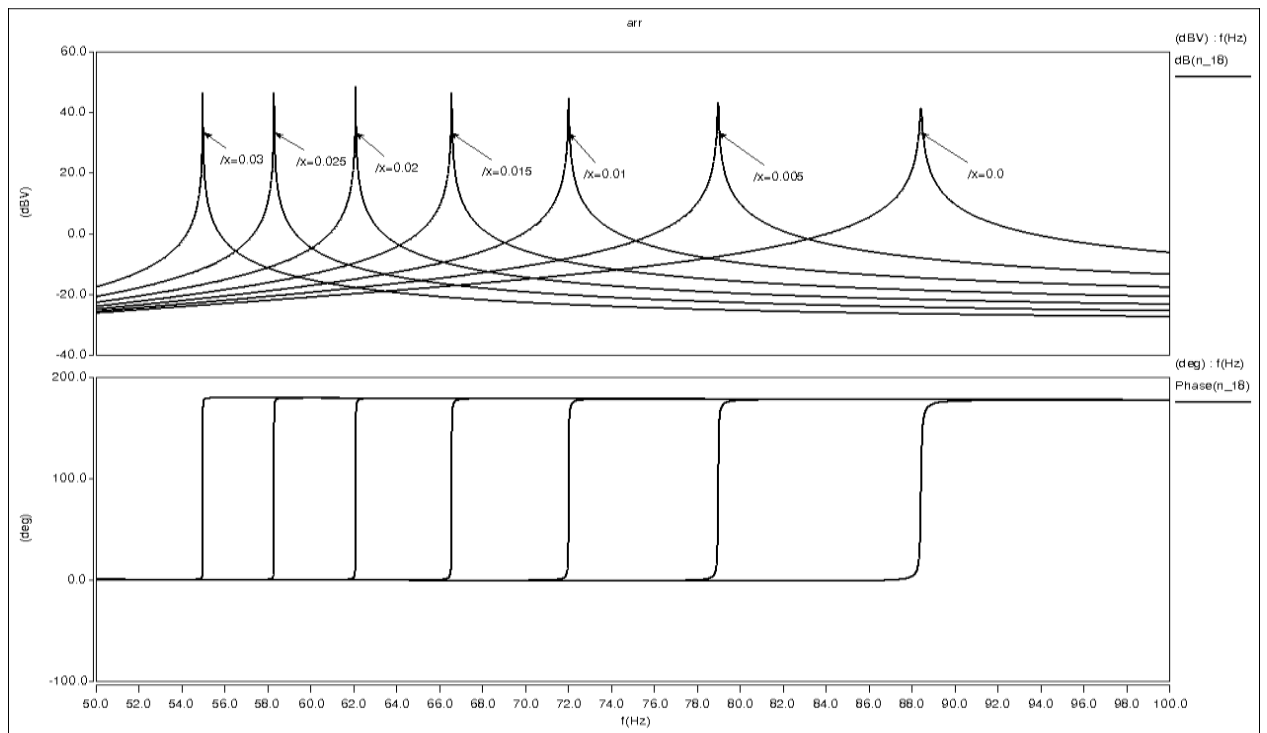

Fig. 10 Frequencies versus voltage output for different PZT position on the Al cantilever beam

\section{Experimental evaluation with practical model}

A flexible Aluminum beam with a clamped end as shown in Fig. 11 is considered in this paper. Two piezoceramic patches are surface bonded on top and bottom of the aluminum beam. Permanent magnet is located at the free end of the beam. The excitation to the system is by means of an electromagnet which is located over the fixed magnet. The dimensions and properties of the beam and piezoceramic patches are given in Table 1 and Table 2.

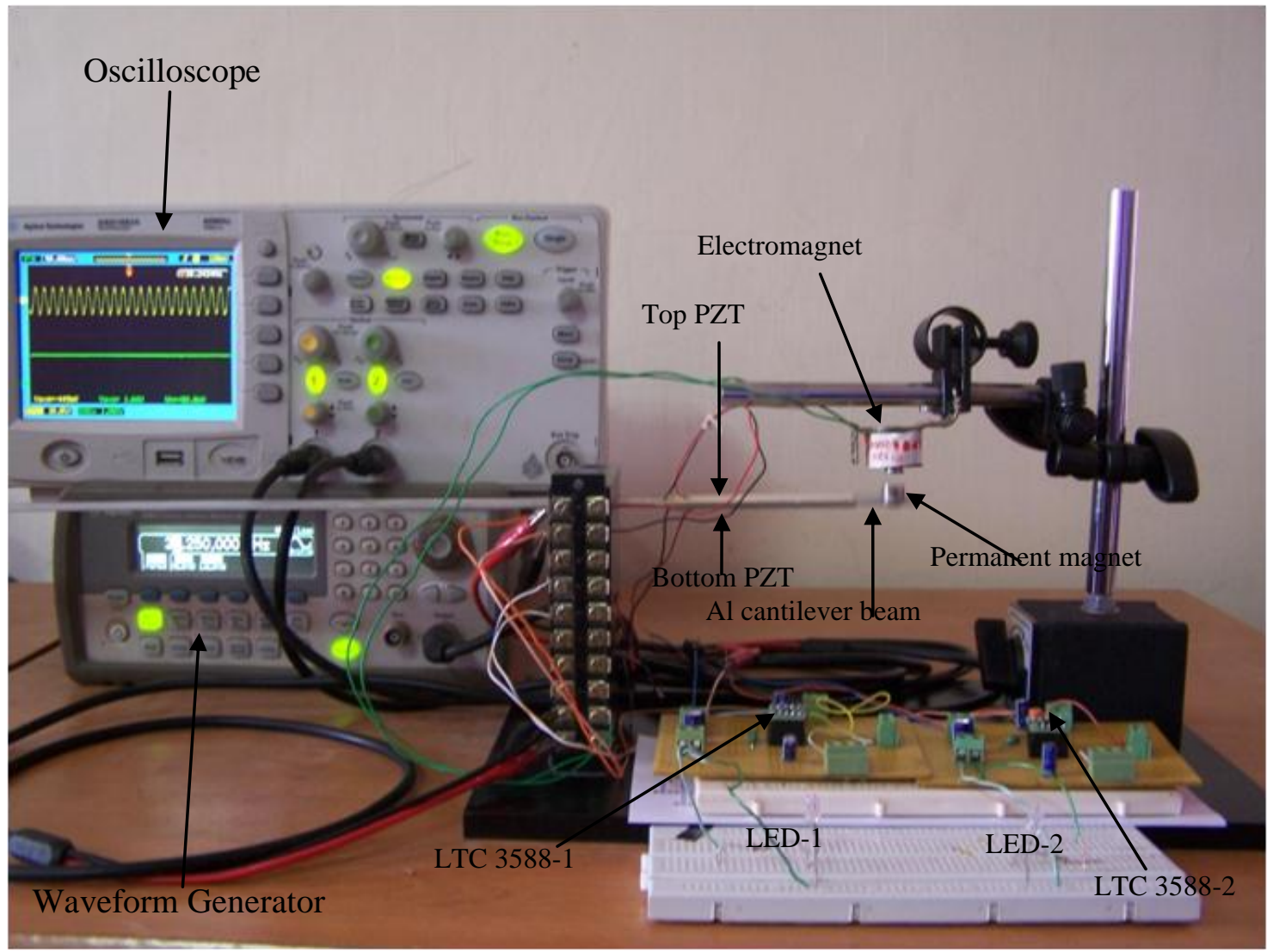

Fig. 11 The image of the experimental setup

The piezoceramic of type SP-5H, which is equivalent to NAVY TYPE VI. The piezoceramics are electroded with fixed-on adherent silver of solderable quality. Electrical contacts to the electrodes are made by soldering. This makes fragile piezoceramics much easier to work with and easier to integrate into the structure. The input to the electromagnetic coil has the frequency which is equal to the natural frequency of the energy harvesting 
structure. Proposed within is the methodology to tune the resonant frequency of the vibration structure via the application of magnetic stiffness/forces using permanent magnets. When a magnetic force is applied to the cantilever, it is forced in to periodic motion at the drive frequency. The strain induced in the cantilever is dependent on the magnitude and frequency of the applied force. The distance between the magnets is limited by the geometrical stiffness in the beam and the magnetic flux density of the magnets. Magnetic contact can be avoided by optimizing the stiffness of the beam and the maximum distance between the magnets. Analytical simulation and experimentation is carried out by changing the input force applied to the Aluminum cantilever beam which is shown in Fig. 12, the results are in close agreement.

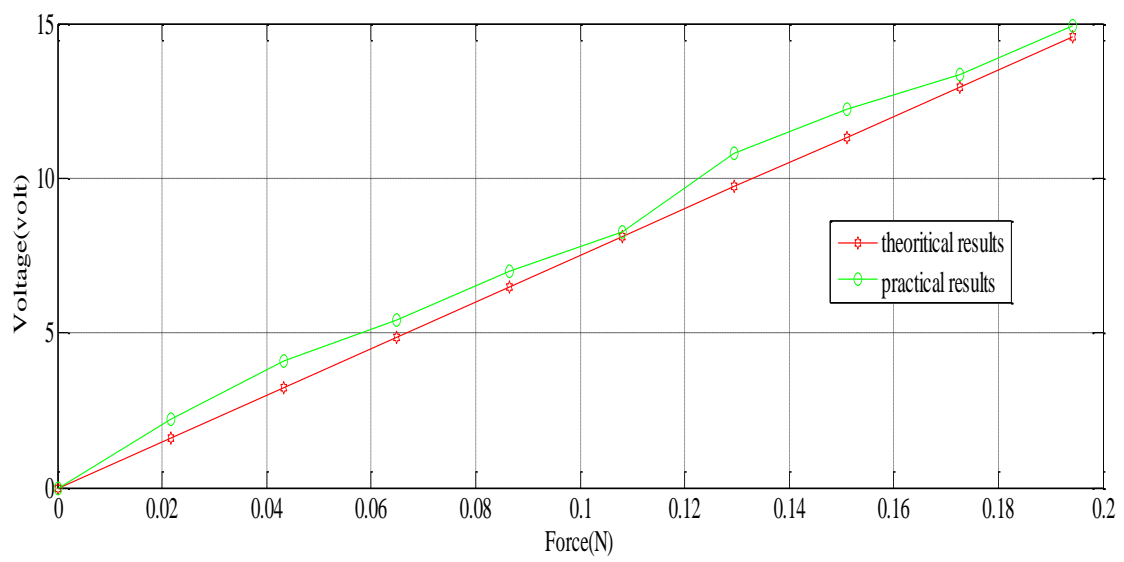

Fig. 12 Force versus voltage of Energy harvester

To manage the energy harvesting and the energy release to the system, the LTC3588-1 piezoelectric energy harvesting power supply integrates a low loss internal bridge rectifier with nanopower high efficiency synchronous buck converter. It uses an efficient optimized energy harvesting algorithm to collect and store energy from high impedance piezoelectric elements, which can have short-circuited currents on the order of tens of micro amps. An ultra quiescent current under voltage lockout (UVLO) mode with a wide hysteresis window allows charge to accumulate on an input capacitor until the buck converter can efficient transfer a portion of the stored charge to the output. In regulation, the LTC 3588-1 enters a sleep state in which both input and output quiescent currents are minimal. The buck converter turns on and off as needed to maintain voltage regulation and bleeds off any excess power via an internal shunt regulator. The LTC 3588-1 has a feature of selecting of 4 different output voltages ranging from $1.8 \mathrm{~V}$ to $3.6 \mathrm{~V}$ and up to $100 \mathrm{~mA}$ of continuous output current and LTC $3588-2$ has range of $3.45 \mathrm{~V}$ to $5 \mathrm{~V}$. The output voltage is selected depending upon the application requirements.

The output response of the energy harvesting IC LTC3588-1 for $5 \mathrm{~V}_{\mathrm{PP}}$ selection is shown in Fig. 13(a) which shows average DC output voltage of $4.96 \mathrm{~V}$. To observe the load characteristics both the piezoelectrics are connected to LTC 3588 at $3.6 \mathrm{~V}$ range selection. Output of one piezoelectric is used to drive the load (LED) and the other output without load are shown in Fig. 13(b). When load is connected the voltage drops down to $1.23 \mathrm{~V}$ from $3.52 \mathrm{~V}$. The load current of $52 \mu \mathrm{A}$ is measured by Agilent $3458 \mathrm{~A} 81 / 2$ digital multimeter and the power consumption is around $64 \mu \mathrm{W}$.

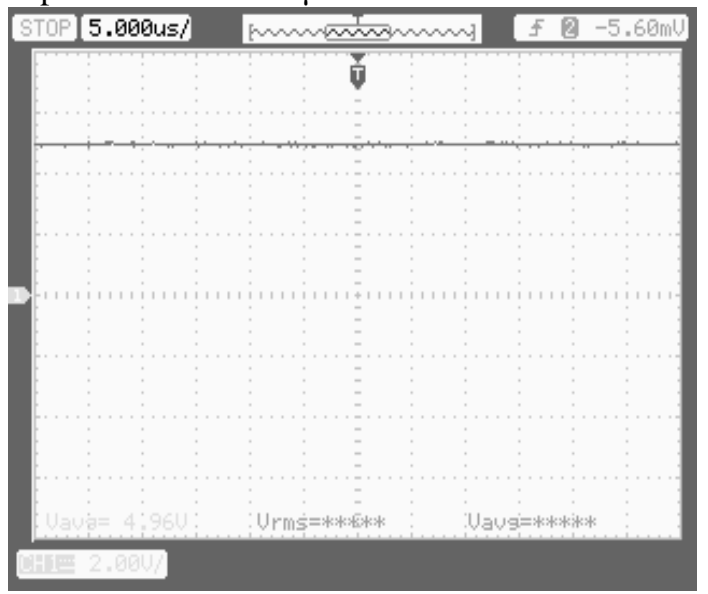

(a)

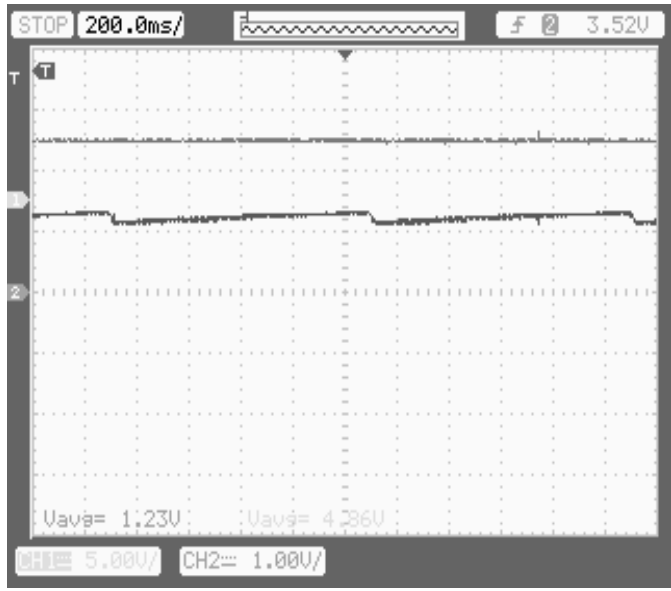

(b)

Fig. 13(a) Study state response from the IC output with $\mathrm{D} 0=1$ and $\mathrm{D} 1=1(\mathrm{I} / \mathrm{P}=5 \mathrm{Vpp})$

(b) Study state response of two sensors one is connected to load other under no load 


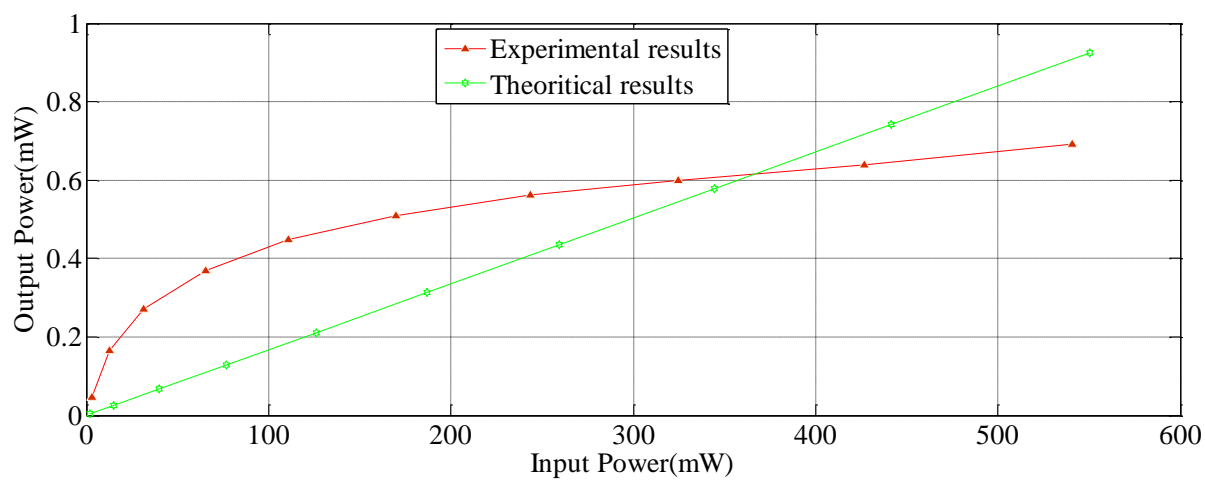

Fig. 14 The input and output characteristics of piezoelectric energy harvester

The input and output power characteristics of the piezoelectric energy harvester are shown in Fig. 14. From the experimental results it is found that the output power remains almost constant after a certain point depending upon the PZT dimension.

\section{Conclusions}

Genetic Algorithm based optimization approach for the design of Energy harvester which shows great potential and the possibility for enhanced energy recovery from ambient vibrations. Numerical analysis is also carried out using FEM design and simulation software COVENTORWARE to decide the optimum PZT position on the Aluminum cantilever beam for maximum output power. For the designed structure the optimum position for PZT on beam is found to be $2 \mathrm{~cm}$ from the fixed end. Experiments were carried out to support numerical analysis. The harvested AC power is converted into usable DC power by Piezoelectric Energy Harvesting Power Supply device LTC3588. The output current of $52 \mu \mathrm{A}$ is measured with $10 \mathrm{ohm}$ resistance LED as a load and the power produced is around $64 \mu \mathrm{W}$ from single piezoelectric. MEMS sensors, hearing aids and other low power wireless nodes can be powered by combining the output power from both the piezoelectric.

\section{Acknowledgements}

The authors would like to acknowledge the support from the Department of Science and Technology from Indian government, under the grant No. 100/IFD/6520/2010-11.

\section{References}

[1] Marco Ferrari, Vittorio Ferrari, Michele Guizzetti and Daniele Marioli, "An autonomous battery less sensor module powered by piezoelectric energy harvesting with RF transmission of multiple measurement signals", Smart materials and Structures.18(2009), pp.1-9.

[2] Vinod R Challa, M G Prasad and Frank T Fisher, "Towards an autonomous self-tuning vibration energy harvesting device for wireless sensor network applications", Smart materials and Structures. 20 (2011), pp.1-11.

[3] Ramadass Y.K. and A. P. Chandrakasan, "An efficient piezoelectric energy harvesting interface circuit using a bias-flip rectifier and shared inductor", IEEE J. Solid State Circuits, vol. 45 (2010), no. 1, pp. 189-204.

[4] Anton S.R. and H.A. Sodano, "A review of power harvesting using piezoelectric materials (2003-2006)", Smart materials and Structures. 16 (2007), pp. R1-R21.

[5] N.G. Stephen, "On energy harvesting from ambient vibration”, Journal of Sound and Vibration 293 (2006) 409-425.

[6] Thiago Seuaciuc-Osorio, Mohammed F. Daqaq, "Energy harvesting under excitations of time-varying frequency", Journal of Sound and Vibration 329(2010)2497-2515.

[7] Khaligh A., P. Zeng, and C. Zheng, "Kinetic energy harvesting using piezoelectric and electromagnetic technologies-State of the art", IEEE Transactions, Industrial Electronics, vol. 57 (2010), no. 3, pp. 850-860.

[8] Lei Wang and F G Yuan, "Vibration energy harvesting by magnetostrictive material", Smart materials and Structures. 17 (2008), pp.1-14.

[9] Steven R Anton1 and Henry A Sodano, "A review of power harvesting using piezoelectric materials", Smart materials and Structures.16 (2007), pp. R1-R21.

[10] Y.K.Tan Y.K. and S. Panda, "Optimized wind energy harvesting system using resistance emulator and active rectifier for wireless sensor nodes", IEEE Transactions, Power Electronics, vol. 26 (2011), no. 1, pp. 38-50.

[11] Jamil M.Renno, Mohammed F.Daqaq, Daniel J.Inman, "On the optimal energy harvesting from a vibration source", Journal of Sound and Vibration 320(2009)386-405.

[12] B.P. Mann, B.A.Owens, "Investigations of a nonlinear energy harvester with a bistable potential well", Journal of Sound and Vibration329 (2010)1215-1226.

[13] Timothy G S Overly, Gyuhae Park, Kevin M Farinholtand Charles R Farrar, "Development of an extremely compact impedancebased wireless sensing device", Smart materials and Structures.17 (2008), pp.1-9.

[14] Hui Shen, Jinhao Qiu, Hongli Ji, Kongjun Zhu and Marco Balsi, "Enhanced synchronized switch harvesting: a new energy harvesting scheme for efficient energy extraction", Smart materials and Structures.19 (2010), pp.1-14.

[15] Yabin Liao and Henry A Sodano, "Optimal parameters and power characteristics of piezoelectric energy harvesters with an $R C$ circuit”, Smart materials and Structures.18 (2009), pp.1-11.

[16] Ji-Tzuoh Lin, Barclay Lee and Bruce Alphenaar, "The magnetic coupling of a piezoelectric cantilever for enhanced energy harvesting efficiency", Smart materials and Structures.19 (2010), pp.1-7. 
[17] Alex Phipps and Toshikazu Nishida, "System modeling of piezoelectric energy harvesters", IEEE Transactions, Power Electronics, vol. 27 (2012), no. 2, pp. 790-802.

[18] Kuok H. Mak, Stewart McWilliam, Atanas A. Popov, Colin H.J. Fox, "Performance of a cantilever piezoelectric energy harvester impacting a bump stop", Journal of Sound and Vibration 330(2011)6184-6202.

[19] C.R. McInnesa, D.G. Gorman, M.P. Cartmell, "Enhanced vibrational energy harvesting using nonlinear stochastic resonance", Journal of Sound and Vibration 318 (2008) 655-662.

[20] SaberMohammadi and Akram Khodayari, "Damping Analyses of Structural Vibrations and Shunted Piezoelectric Transducers", Smart Materials Research, Hindawi Publishing Corporation, Volume 2012, Article ID 431790.

[21] Rohan Dayal, Suman Dwari, Leila Parsa, "A new design for vibration-based electromagnetic energy harvesting systems using coil inductance of micro generator”, IEEE Transactions on industry applications, vol.47 (2011), no.2, pp. 820-830.

[22] Soobum Lee and Byeng D. Youn, "A new piezoelectric energy harvesting design concept: Multimodal energy harvesting skin", IEEE Transactions on Ultrasonics, Ferroelectrics, and Frequency Control, vol. 58 (2011), no. 3, pp. 629-645.

[23] Xiaotian Li, Mingsen Guo, and Shuxiang Dong, "A flex-compressive-mode piezoelectric transducer for mechanical vibration/strain energy harvesting”, IEEE Transactions on Ultrasonics, Ferroelectrics, and Frequency Control, vol. 58 (2011), no. 4, pp. 698-703.

[24] Amin M. Karami, Onur Bilgen, Daniel J. Inman, and Michael I. Friswell, "Experimental and analytical parametric study of singlecrystal unimorph beams for vibration energy harvesting", IEEE Transactions on Ultrasonics, Ferroelectrics, and Frequency Control, vol. 58 (2011), no. 7, pp. 1508-1520.

[25] Cheng Luo, Heath F. Hofmann, "Wideband energy harvesting for piezoelectric devices with linear resonant behavior", IEEE Transactions on Ultrasonics, Ferroelectrics, and Frequency Control, vol. 58(2011), no. 7, pp. 1294-1301.

[26] Carlos De Marqui Junior, Alper Erturk, Daniel J. Inman, “An electromechanical finite element model for piezoelectric energy harvester plates", Journal of Sound and Vibration 327(2009)9-25.

[27] John M.Youngsman, Tim Luedeman, Dylan J. Morris, Michael J. Anderson, David F. Bahr, "A model for an extensional mode resonator used as a frequency-adjustable vibration energy harvester", Journal of Sound and Vibration 329(2010)277-288.

[28] A. Erturk, D. J. Inman, "Broadband piezoelectric power generation on high-energy orbits of the bistable Duffing oscillator with electromechanical coupling”, Journal of Sound and Vibration 330(2011)2339-2353.

[29] Shashank Priya, Daniel J. Inman (2009), "Energy Harvesting Technologies", New York, NY: Springer. 